\title{
Assessment of Post-Resuscitation Intestinal Injury and Timing of Bacterial Translocation in Swine Anaesthetized With Propofol-Based Total Intravenous Anaesthesia
}

Andreas Tassopoulos ${ }^{1}$, Athanasios Chalkias ${ }^{2}$, Apostolos Papalois ${ }^{3}$, Paraskevi Karlovasiti ${ }^{4}$, Jacopo Sergio Antonio Zanda ${ }^{5}$, Stefanos Chatzidakis ${ }^{6}$, Maria Gazouli ${ }^{7}$, Nicoletta Iacovidou ${ }^{8}$, Daniela Fanni ${ }^{9}$, Theodoros Xanthos 10

1. Department of Cardiology, Red Cross General Hospital, Athens, GRC 2. Department of Anesthesiology, University of Thessaly, School of Health Sciences, Faculty of Medicine, Larisa, GRC 3. Translational Research and Training, ELPEN Research \& Experimental Center, Athens, GRC 4. Department of Biopathology - Microbiology and Biochemistry, Medical School, National and Kapodistrian University of Athens, Athens, GRC 5. Division of Pathology, Department of Surgical Sciences, University of Cagliari, Cagliari, ITA 6. Department of Medicine, European University Cyprus, School of Medicine, Nicosia, CYP 7. Laboratory of Biology, Medical School, National and Kapodistrian University of Athens, Athens, GRC 8. Department of Neonatology, Medical School, National and Kapodistrian University of Athens, Athens, GRC 9. Division of Pathology, Department of Medical Sciences and Public Health, University of Cagliari, Cagliari, ITA 10. Department of Physiology and Pathophysiology, European University Cyprus, School of Medicine, Nicosia, CYP

Corresponding author: Athanasios Chalkias, thanoschalkias@yahoo.gr

\section{Abstract \\ Introduction and objectives}

Bacterial translocation (BT) is the passage of viable bacteria or endotoxins from the gastrointestinal lumen to extra-luminal tissues and is usually observed after intestinal ischaemia-reperfusion injury. The aim of this study was to investigate post-resuscitation BT after cardiac arrest and resuscitation in a swine anaesthetized with propofol-based total intravenous anaesthesia.

\section{Materials and methods}

Eighteen female Landrace/Large White piglets were randomly divided into control (CON), cardiac arrest (CA) and cardiac arrest-cardiopulmonary resuscitation (CA-CPR) groups. In the CON group, the animals were only monitored for two hours. In the CA group, the animals were not resuscitated and underwent necropsy immediately after cardiac arrest. In the CA-CPR group, the animals were resuscitated until the return of spontaneous circulation (ROSC) and were monitored for two hours. The animals of the CON and CA-CPR groups underwent necropsy 24 hours later. Bacterial translocation was assessed by blood and tissue cultures and endotoxin measurement in the portal and systemic circulation. Malondialdehyde content calculation and histological analysis of the intestine were performed in order to estimate ischemia and reperfusion (I/R) tissue damage.

Received 06/25/2020

Review began 06/26/2020

Review ended 08/25/2020

Published 09/10/2020

(c) Copyright 2020

Tassopoulos et al. This is an open access article distributed under the terms of the Creative Commons Attribution License CC-BY 4.0., which permits unrestricted use, distribution, and reproduction in any medium, provided the original author and source are credited.

\section{Results}

Malondialdehyde content, an indicator of oxidative stress, was significantly higher in the CA-CPR group compared to the CA in homogenized ileum $(\mathrm{p}=0.016)$. Malondialdehyde content in homogenized colon revealed significantly higher levels in the CA-CPR group compared to the CON ( $p=0.004)$ and the CA group $(\mathrm{p}=0.016)$. We found significantly higher levels of portal endotoxin in the CA-CPR group compared to the CON ( $\mathrm{p}=0.026$ ) and the CA group ( $\mathrm{p}=0.026$ ). The number of positive mesenteric lymph nodes cultures for $E$. coli was greater in the CA-CPR group, followed by the CA and CON groups, although the difference was not significant (67\%, 33\%, and 33\%, respectively; $\mathrm{p}=0.407)$.

\section{Conclusions}

Malondialdehyde content and portal endotoxin levels do not increase during the cardiac arrest interval, but only after CPR and ROSC. Although the number of positive MLNs cultures was greater in the CA-CPR animals, no statistically significant differences were observed between the three groups due to the short monitoring period.

Categories: Anesthesiology, Emergency Medicine

Keywords: bacterial translocation, propofol, cardiac arrest, post-resuscitation period, reperfusion

\section{Introduction}

Cardiac arrest is a medical emergency with more than one million cases per year and dismal prognosis. Even 
if return of spontaneous circulation (ROSC) is achieved, survival to hospital discharge ranges from $9.4 \%$ for out-of-hospital cardiac arrest (OHCA) to $22.4 \%$ for in-hospital cardiac arrest [1-3]. In addition, the risk for post-resuscitation septic complications is high; more than one-third of OHCA victims become bacteremic within the first 12 hours after ROSC, while bacteraemia or other confirmed source of infection may aggravate neurologic outcomes and increase mortality [4].

Bacterial translocation (BT) is the passage of viable bacteria or endotoxins from the gastrointestinal lumen, through the mucosal epithelium, to extra-luminal tissues, such as the mesenteric lymph nodes (MLNs) and other distant organs [5-7]. This phenomenon is usually observed after intestinal ischemia-reperfusion (I/R) injury, but the timing and the associated pathophysiological mechanisms have been poorly investigated after cardiac arrest and resuscitation.

The primary aim of this study was to investigate when BT occurs after the onset of cardiac arrest in animals anaesthetized with propofol-based total intravenous anaesthesia. Secondary aims were the assessment of intestinal oxidative stress and intestinal injury post-cardiac arrest.

\section{Materials And Methods}

The study protocol was approved by the Greek General Directorate of Veterinary Services (license No 4978/16-10-2015) and was conducted in accordance to the Greek legislation regarding ethical and experimental procedures.

\section{Animal preparation}

Study subjects were 18 female Landrace/Large-White piglets, aged 10-15 weeks, with an average weight of $19 \pm 2 \mathrm{~kg}$. The animals were purchased from the same breeder (Validakis, Koropi, Greece) and were transported to the research facility (Experimental Research Center, ELPEN, European Ref No. EL 09 BIO 03) one week before experimentation. Prior to the experimental procedure, the animals were fasted overnight but had free access to water [8]. All animals were examined by a veterinarian on the day of the experimentation and were found healthy.

Animals were premedicated with intramuscular injections of $10 \mathrm{mg} / \mathrm{kg}$ ketamine hydrochloride (Merial, Lyon, France), $0.5 \mathrm{mg} / \mathrm{kg}$ midazolam (Roche, Athens, Greece), and $0.05 \mathrm{mg} / \mathrm{kg}$ atropine sulphate (Demo, Athens, Greece), as previously described [9]. The animals were subsequently transported to the operation research facility and intravascular access through the auricular veins was obtained. Anaesthesia was induced by an intravenous bolus dose of $2 \mathrm{mg} / \mathrm{kg}$ propofol (Diprivan 1\% w/v; Astra Zeneca, Luton, United Kingdom) and $2 \mu \mathrm{g} / \mathrm{kg}$ fentanyl (Janssen Pharmaceutica, Beerse, Belgium) [10]. Whilst spontaneously breathing but anaesthetized, the animals were intubated with an endotracheal tube (Portex, $4.5 \mathrm{~mm}$ ID; Mallinckrodt Medical, Athlone, Ireland) and were immobilized in the supine position on a surgical table. Additional 1 $\mathrm{mg} / \mathrm{kg}$ propofol, $0.15 \mathrm{mg} / \mathrm{kg}$ cis-atracurium (Nimbex $2 \mathrm{mg} / \mathrm{mL}$; GlaxoSmithKline, Athens, Greece), and 0.01 $\mathrm{mg} / \mathrm{kg}$ fentanyl were administered to achieve synchrony with the ventilator. The animals were ventilated with a tidal volume of $10 \mathrm{ml} / \mathrm{kg}$ and $21 \%$ oxygen (Alpha Delta lung ventilator, Siare, Bologna, Italy), while propofol $0.1 \mathrm{mg} / \mathrm{kg} / \mathrm{min}$, cis-atracurium $20 \mu \mathrm{g} / \mathrm{kg} / \mathrm{min}$, and fentanyl $0.6 \mu \mathrm{g} / \mathrm{kg} / \mathrm{min}$ were administered to maintain adequate anaesthetic depth. End-tidal carbon dioxide pressure (ETCO2) was continuously monitored (Tonocap-TC200; Datex Engstrom, Helsinki, Finland) and the respiratory rate was adjusted to maintain ETCO2 between 35 and $45 \mathrm{mmHg}$. Pulse oximetry was monitored throughout the experiment. Electrocardiographic monitoring included leads I, II, III, aVR, aVL, and aVF. The leads were connected to a monitor (Mennen Medical, Envoy; Papapostolou, Athens, Greece) which electronically calculated the heart rate. Arterial blood gases were measured on a blood-gas analyzer (IRMA SL Blood Analysis System, part 436301; Diametrics Medical Inc, Roseville, MN).

For measurement of the aortic pressures, an arterial catheter (model 6523, USCI CR, Bart; Papapostolou) was inserted and forwarded into the descending aorta after surgical preparation of the right internal carotid artery. The systolic (SAP) and diastolic (DAP) arterial pressures were recorded, whereas mean arterial pressure (MAP) was determined by the electronic integration of the aortic blood pressure waveform. The right internal jugular vein was cannulated with a catheter to measure central venous pressure (CVP). The left internal jugular vein was also surgically prepared and a catheter was inserted for fluid administration. Intravascular catheters were attached to pressure transducers that were aligned to the level of the right atrium and were calibrated before their use. This allowed the recording of CVP, right atrial, and arterial pressures. Cardiac output (CO) was measured as the product of time-velocity integral of Doppler transaortic flow, the diameter of the aortic valve, and heart rate, while SVR was calculated using the formula SVR = (MAP - CVP)/CO x 80, as previously described [11].

\section{Experimental procedure}

Baseline data were collected after allowing each animal to stabilize for a 30-minute period. Before the experimental procedure, the animals were randomly divided using closed envelopes into three groups: control (CON, $n=6$ ), cardiac arrest (CA, $n=6$ ) and cardiac arrest and CPR (CA-CPR, $n=6)$. We used three groups to investigate the evolution of intestinal damage during the different stages of cardiac arrest and 
resuscitation. The investigators involved in data recording, data entry, and data analysis were blinded to each animal's allocation.

In the CON group, the animals were subjected only to minor aseptic procedures (instrumentation) and were monitored for two hours. In the CA and CA-CPR group, a 5F flow-directed pacing catheter (Pacel, $100 \mathrm{~cm}$; St Jude Medical, Ladakis, Athens, Greece) was advanced into the apex of the right ventricle and ventricular fibrillation was induced using a 9V ordinary cadmium battery, as previously described [12]. Cardiac arrest was recognized electrocardiographically and confirmed by loss of arterial pulse. Mechanical ventilation and administration of anaesthetics were discontinued simultaneously with the onset of cardiac arrest and the animals were left untreated for eight minutes (representing the average time of EMS arrival in Europe).

In the CA group, the animals were not resuscitated. Blood samples were collected immediately after the eighth minute of cardiac arrest and then the animals underwent necropsy. Tissue samples from MLNs, liver, spleen, and lungs were also collected. In the CA-CPR group, resuscitation was immediately started after the eighth minute of cardiac arrest according to the 2015 European Resuscitation Council guidelines on resuscitation, with ventilation in $100 \%$ oxygen and chest compressions at a rate of 100/min (LUCAS 2 CPR device, Jolife, Lund, Sweden) [13]. Defibrillation was attempted with a $4 \mathrm{~J} / \mathrm{kg}$ monophasic waveform shock delivered between the right infraclavicular area and the cardiac apex (Primedic Defi-B Defibrillator; Metrax $\mathrm{GmbH}$, Rottweil, Germany), while adrenaline was administered at a dose of $0.02 \mathrm{mg} / \mathrm{kg}$ via the marginal auricular vein [8]. Resuscitation efforts continued until ROSC (MAP of at least $60 \mathrm{~mm} \mathrm{Hg}$ for a minimum of five minutes) or when asystole occurred after the tenth minute of CPR. After ROSC, the animals were anaesthetized and monitored for two hours, during which no further resuscitation was attempted.

Subsequently, anaesthesia was discontinued in the CON and CA-CPR groups, all catheters were removed as previously described, and manual ventilation was initiated [14]. Atropine $0.2 \mathrm{mg} / \mathrm{kg}$ followed by neostigmine $0.05 \mathrm{mg} / \mathrm{kg}$ were administered after detection of spontaneous swallowing reflex and extubation was performed when adequate inspiration depth was confirmed. Each animal was then transferred to its house for observation. After 24 hours, the animals were humanely euthanized by an intravenous overdose of pentobarbital and underwent necropsy [15]. Blood and tissue samples were also collected.

\section{Malondialdehyde and endotoxin analysis - Histopathological evaluation}

Using aseptic techniques, tissue samples of 2.0-3.0 cm from terminal ileum and colon were collected and stored in liquid nitrogen at $-70{ }^{\circ} \mathrm{C}$ until analysis. Additional samples of ileum and colon were collected and immediately stored in $10 \%$ formaldehyde. Blood samples from the portal vein and the aorta were collected and immediately stored in endotoxin-free tubes (EndoGrade ${ }^{\circledR}$ Glass Test Tubes, Hyglos/bioMérieux, Bernried, Germany) until analysis. Plasma levels of endotoxin in the portal and the systemic circulation were determined using a commercially available ELISA kit (KIT LS-F15272, Life Span BioSciences, Inc., Seattle, WA, USA). Tissue malondialdehyde (MDA) content in homogenized ileum and colon was measured using a commercially available ELISA kit (KIT EU2577, Wuhan Fine Biological Technology Co., Ltd., Wuhan, China). The degree of intestinal tissue injury was graded from 0 to 5, using a modified Chiu's method, as previously described [16].

\section{Statistical analysis}

During study design, the insufficient data in literature did not allow for power analysis and sample size estimation. Therefore, we were given permission to conduct a pilot study with six animals per group. The pilot data analysis revealed an adequate level of statistical significance and considering the guiding principles underpinning the humane use of animals in scientific research (three Rs), the final sample size included six animals per group $[15,17]$. Data were expressed as mean \pm SD or median \pm IQR for continuous variables. The normality of distributions was assessed using the Kolmogorov-Smirnov test. Categorical variables were compared between groups using the chi-square test, or Fisher's exact test, as required. Comparisons of continuous variables between the three groups were performed using one-way ANOVA and pairwise comparisons were performed using the Bonferroni test. In case of non-normal distributions, the non-parametric Kruskal-Wallis and Mann-Whitney tests were used. Comparisons of continuous data extracted at the predefined time points of the observation period, only for the CON and CA-CPR groups, were performed using independent samples t-test, or Mann-Whitney test in case of violation of normality. All tests were two-tailed and a value of $\mathrm{p}<0.05$ was considered significant. Statistical analysis was performed using SPSS version 21.0 (IBM Corp, Armonk, NY, USA).

\section{Results}

All animals in the CA-CPR group were successfully resuscitated.

\section{Haemodynamic and metabolic parameters}

No statistically significant differences were observed in baseline haemodynamic and metabolic parameters between the three groups (Table 1). 


\section{Cureus}

\begin{tabular}{|c|c|c|c|c|}
\hline \multirow{2}{*}{ Measurement } & CON & CA & CA-CPR & \multirow{2}{*}{ p-value } \\
\hline & Mean $\pm S D$ & Mean $\pm S D$ & Mean $\pm S D$ & \\
\hline Heart rate (beats/min) & $106.83 \pm 11.53$ & $107.00 \pm 5.73$ & $96.33 \pm 14.99$ & 0.212 \\
\hline Systolic aortic pressure $(\mathrm{mmHg})$ & $115.50 \pm 7.4$ & $116.83 \pm 10.34$ & $115.17 \pm 10.74$ & 0.951 \\
\hline Diastolic aortic pressure $(\mathrm{mmHg})$ & $92.17 \pm 5.78$ & $94.00 \pm 10.86$ & $89.67 \pm 10.09$ & 0.720 \\
\hline Systolic right atrial pressure (mmHg) & $12.00 \pm 4.43$ & $10.17 \pm 3.06$ & $13.50 \pm 3.02$ & 0.297 \\
\hline Diastolic right atrial pressure $(\mathrm{mmHg})$ & $6.83 \pm 2.48$ & $5.33 \pm 2.42$ & $8.33 \pm 2.50$ & 0.144 \\
\hline Cardiac output (L/min) & $5.53 \pm 0.61$ & $4.93 \pm 1.00$ & $4.90 \pm 0.99$ & 0.399 \\
\hline $\mathrm{pH}$ & $7.44 \pm 0.05$ & $7.39 \pm 0.03$ & $7.41 \pm 0.04$ & 0.160 \\
\hline $\mathrm{pO}_{2}(\mathrm{mmHg})$ & $128.17 \pm 19.05$ & $111.33 \pm 17.37$ & $119.00 \pm 16.75$ & 0.287 \\
\hline $\mathrm{pCO}_{2}(\mathrm{mmHg})$ & $42.5 \pm 4.04$ & $45.83 \pm 2.40$ & $42.67 \pm 4.27$ & 0.240 \\
\hline $\mathrm{HCO}_{3}(\mathrm{mmol} / \mathrm{L})$ & $27.68 \pm 2.87$ & $26.50 \pm 1.63$ & $26.20 \pm 2.25$ & 0.515 \\
\hline Lactate $(\mathrm{mmol} / \mathrm{L})$ & $0.51 \pm 0.32$ & $0.58 \pm 0.15$ & $0.97 \pm 0.91$ & 0.348 \\
\hline
\end{tabular}

\section{TABLE 1: Baseline hemodynamic and metabolic parameters of the study}

CON, control group; CA, cardiac arrest group; CA-CPR, cardiac arrest and cardiopulmonary resuscitation group.

All animals in the CA-CPR group restored ROSC after 6.3 $\pm 2.1 \mathrm{~min}$ of CPR and a mean adrenaline dose of $0.5 \pm 0.17$.

Statistically significant differences were observed in $\mathrm{pH}(\mathrm{p}=0.002), \mathrm{HCO} 3(\mathrm{p}=0.003)$ and lactate $(\mathrm{p}=0.008)$ between the CON and the CA-CPR group one hour after ROSC. Two hours after ROSC, $\mathrm{pH}$ and HCO3 levels remained significantly lower $(\mathrm{p}=0.016$ and $\mathrm{p}=0.007$, respectively), whereas lactate concentration remained significantly higher $(\mathrm{p}=0.046)$ in the CA-CPR group (Table 2). 


\section{Cureus}

\begin{tabular}{|c|c|c|c|c|c|c|}
\hline \multirow{3}{*}{ Measurement } & \multicolumn{3}{|l|}{1 hour } & \multicolumn{3}{|l|}{2 hours } \\
\hline & CON & CA-CPR & & CON & CA-CPR & \\
\hline & Mean \pm SD & Mean $\pm S D$ & & Mean \pm SD & Mean \pm SD & \\
\hline Heart rate (beats/min) & $101.67 \pm 9.50$ & $110.50 \pm 13.20$ & 0.213 & $99.83 \pm 8.26$ & $103.17 \pm 12.40$ & 0.596 \\
\hline Systolic aortic pressure $(\mathrm{mmHg})$ & $112.83 \pm 13.4438$ & $103.17 \pm 7.76$ & 0.158 & $112.00 \pm 18.49$ & $117.50 \pm 7.77$ & 0.524 \\
\hline Diastolic aortic pressure (mmHg) & $86.17 \pm 10.70$ & $82.50 \pm 6.66$ & 0.492 & $82.33 \pm 16.37$ & $93.83 \pm 7.94$ & 0.164 \\
\hline Systolic right atrial pressure (mmHg) & $11.50 \pm 2.43$ & $13.17 \pm 2.79$ & 0.295 & $12.17 \pm 2.56$ & $13.50 \pm 3.51$ & 0.469 \\
\hline Diastolic right atrial pressure $(\mathrm{mmHg})$ & $7.67 \pm 2.58$ & $8.17 \pm 2.48$ & 0.740 & $7.83 \pm 2.40$ & $8.33 \pm 3.39$ & 0.774 \\
\hline Cardiac output (L/min) & $6.05 \pm 1.48$ & $5.55 \pm 0.82$ & 0.485 & $6.25 \pm 1.41$ & $4.83 \pm 0.79$ & 0.064 \\
\hline $\mathrm{pH}$ & $7.4 \pm 0.05$ & $7.28 \pm 0.06$ & 0.002 & $7.41 \pm 0.05$ & $7.32 \pm 0.06$ & 0.016 \\
\hline $\mathrm{pO}_{2}(\mathrm{mmHg})$ & $128.17 \pm 27.86$ & $108.33 \pm 19.75$ & 0.185 & $121.17 \pm 29.67$ & $113.17 \pm 28.43$ & 0.644 \\
\hline $\mathrm{pCO}_{2}(\mathrm{mmHg})$ & $43.17 \pm 4.40$ & $40.50 \pm 2.59$ & 0.229 & $42.33 \pm 3.56$ & $39.50 \pm 2.26$ & 0.131 \\
\hline $\mathrm{HCO}_{3}(\mathrm{mmol} / \mathrm{L})$ & $26.05 \pm 3.94$ & $18.75 \pm 2.36$ & 0.003 & $26.35 \pm 3.44$ & $19.78 \pm 3.20$ & 0.007 \\
\hline Lactate $(\mathrm{mmol} / \mathrm{L})$ & $0.70 \pm 0.35$ & $3.27 \pm 1.85$ & 0.008 & $0.59 \pm 0.47$ & $2.21 \pm 1.50$ & 0.046 \\
\hline
\end{tabular}

TABLE 2: Hemodynamic and metabolic parameters in the CON and CA-CPR groups during the monitoring period

CON: control group; CA: cardiac arrest group; CA-CPR: cardiac arrest and cardiopulmonary resuscitation group.

\section{Intestinal mucosal injury and malondialdehyde content}

Although histological analysis revealed mild intestinal damage in the CA and CA-CPR groups, there was no statistically significant difference in intestinal mucosal injury score between the three groups $(\mathrm{p}=0.504)$ (Table 3, Fig. 1).

\begin{tabular}{|c|c|c|c|c|c|c|c|}
\hline & \multicolumn{2}{|l|}{ CON } & \multicolumn{2}{|l|}{ CA } & \multicolumn{2}{|l|}{ CA-CPR } & \multirow{2}{*}{ p-value } \\
\hline & Median & IQR & Median & IQR & Median & IQR & \\
\hline MDA - lleum (ng/ml) & 23.02 & 4.51 & 20.99 & 7.77 & $27.46^{\mathrm{a}}$ & 4.53 & 0.042 \\
\hline MDA - Colon (ng/ml) & $9.73^{b}$ & 8.06 & $10.56^{c}$ & 8.19 & 18.82 & 10.36 & 0.006 \\
\hline Mucosal Injury Score & 2.00 & 2.50 & 3.00 & 2.50 & 3.00 & 1.00 & 0.504 \\
\hline
\end{tabular}

TABLE 3: Comparison of malondialdehyde content and mucosal injury between the three groups

${ }^{a} p=0.016$ vs $C A ;{ }^{b} p=0.004$ vs $C A-C P R ;{ }^{c} p=0.016$ vs $C A-C P R$

MDA: malondialdehyde; CON: control group; CA: cardiac arrest group; CA-CPR: cardiac arrest and cardiopulmonary resuscitation group. 


\section{Cureus}
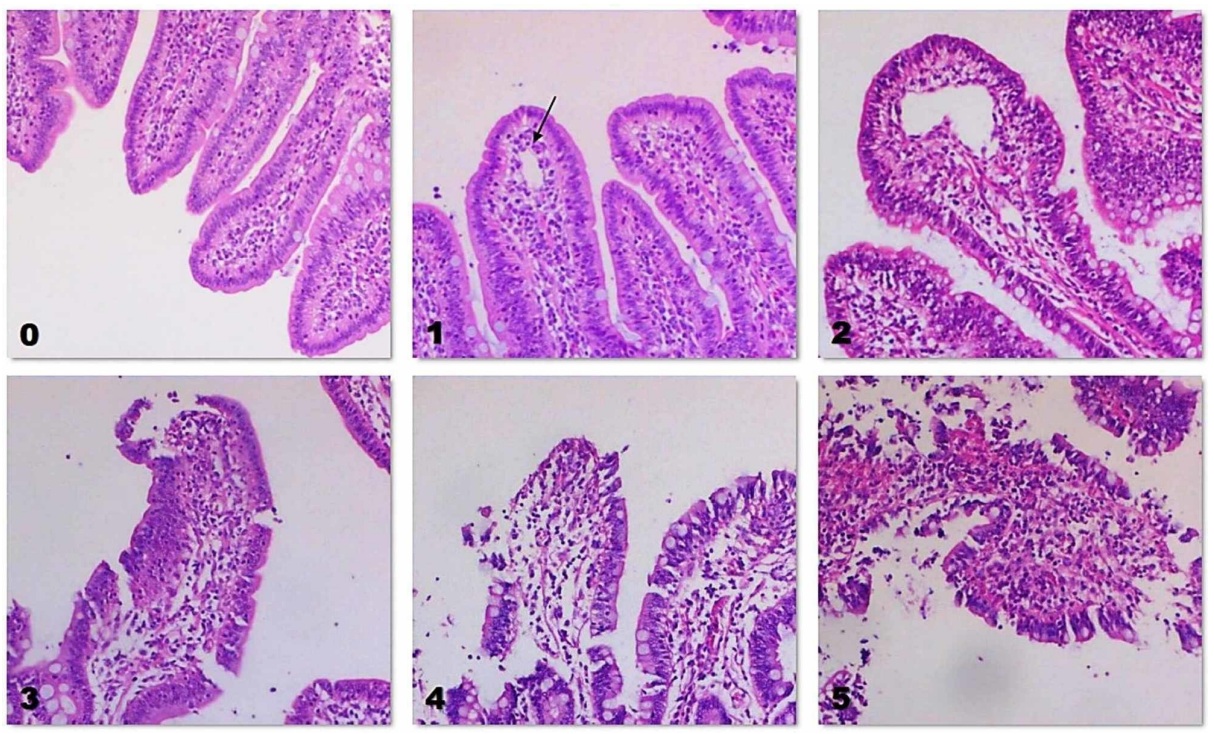

FIGURE 1: Intestinal mucosal injury in our study. Magnification 200x.

0: Normal villi - CON group.

1: Development of the sub-epithelial Gruenhagen's space (black arrow) with some capillary congestion CON group.

2: Extension of the sub-epithelial space with moderate lifting of the epithelial layer from the lamina propria CA group.

3: Massive epithelial lifting with a few denuded villi - CA group

4: Denuded villi with exposure of dilatated capillaries - CA-CPR group.

5: Digestion and disintegration of lamina propria, hemorrhage and ulceration - CA-CPR group.

CON: Control; CA: cardiac arrest; CA-CPR: cardiac arrest-cardiopulmonary resuscitation

We found no statistically significant difference in the MDA content of the homogenized ileum $(\mathrm{p}=0.337)$ and colon ( $\mathrm{p}=0.337$ ) between the CA and CON groups. Malondialdehyde content in homogenized ileum was significantly higher in the CA-CPR group compared to the CA group ( $\mathrm{p}=0.016)$, but not compared to the CON group ( $\mathrm{p}=0.109$ ). Also, MDA measurements in homogenized colon revealed significantly higher levels in the CA-CPR group compared to the $\mathrm{CON}(\mathrm{p}=0.004)$ and the CA group $(\mathrm{p}=0.016)$ (Figure 2$)$. 


\section{Cureus}

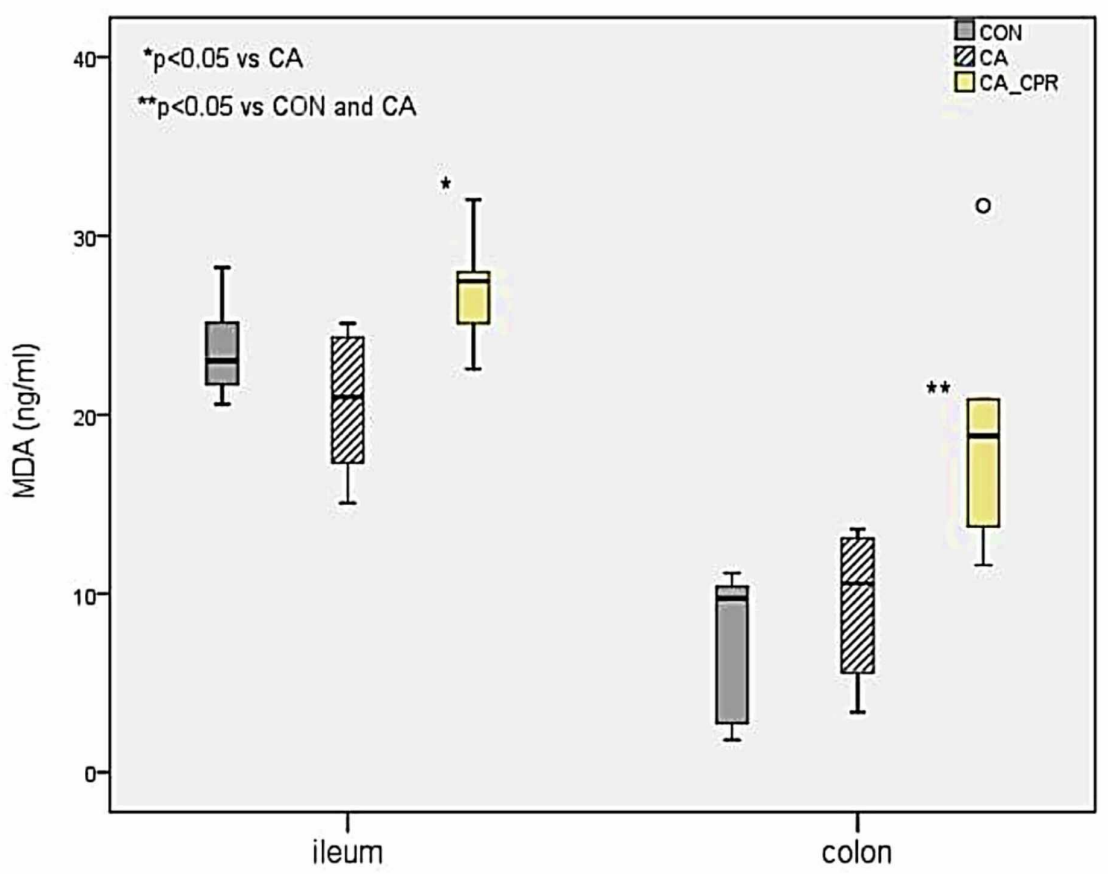

FIGURE 2: Malondialdehyde concentration in homogenized ileum-colon.

CON: control group; CA: cardiac arrest group; CA-CPR: cardiac arrest and cardiopulmonary resuscitation group.

\section{Endotoxin levels}

No statistically significant differences were observed in endotoxin levels in the systemic circulation between the three groups ( $\mathrm{p}=0.628)$. On the contrary, measurements in the portal circulation revealed significantly higher levels of endotoxin in the CA-CPR group compared to the CA $(p=0.026)$ and the CON ( $p=0.026)$ group. However, there was no significant difference in portal endotoxin levels between the CA and the CON group $(\mathrm{p}=0.240)$ (Figure 3).

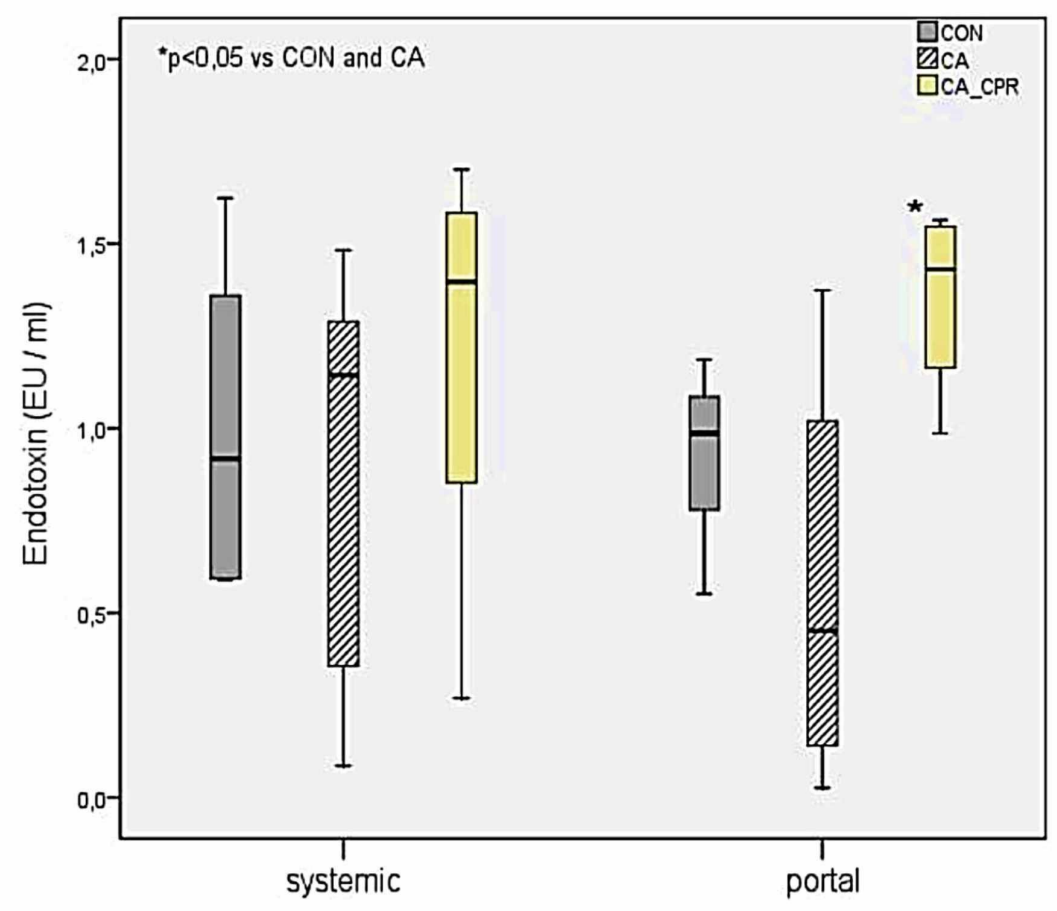

FIGURE 3: Endotoxin levels in systemic and portal circulation. 


\section{Blood and tissue cultures}

The number of positive MLNs cultures for $E$. coli was greater in the CA-CPR group, followed by the CA and CON groups, although the difference was not significant $(67 \%, 33 \%$, and $33 \%$, respectively; $\mathrm{p}=0.407$ ). All other blood and tissue cultures collected at 24 hours post-ROSC were not indicative of BT.

\section{Discussion}

Despite advances in resuscitation science and the increasing evidence on the pathophysiology of postresuscitation syndrome, the time at which BT occurs after cardiac arrest remains unknown. In this study, we found that CA-CPR animals had significantly higher levels of endotoxin in portal circulation compared to CA and CON animals. In addition, the CA-CPR animals had more positive MLNs cultures compared to the other groups, although the difference was not statistically significant. To our knowledge, this is the first study presenting direct evidence regarding the timing of BT after cardiac arrest.

Malondialdehyde, the end product of fatty acid peroxidation, is a good indicator of oxidative injury and the degree of lipid peroxidation and oxidative stress [18]. In our study, we did not find significant differences in the MDA content of the homogenized ileum and colon between the CA and CON groups. Considering that our animals were healthy without any comorbidity, we conclude that intestinal ischaemia alone could not increase oxidative stress during the eight-minute cardiac arrest interval, indicating significant intestinal physiological reserves possibly due to preserved microcirculatory autoregulation [19]. After the onset of $\mathrm{CPR}$, the gut receives a small amount of the compression-related cardiac output (less than 5\%) and thus the major intestinal disturbance during CPR continues to be ischaemia rather than reperfusion [5]. However, even this small amount of oxygenated blood may be capable for inducing reperfusion injury, which is significantly exacerbated after ROSC [20]. This was also evident in the CA-CPR group, in which intestinal reperfusion increased inflammation and the oxidative stress, resulting in a higher MDA content in these animals.

Despite the significant differences in the MDA content of our animals, several authors have reported that treatment with propofol decreases intestinal MDA levels [16]. Therefore, propofol may have exerted protection against oxidative stress, resulting in lower MDA levels in study [16]. In another animal study, pretreatment with a sedative dose of propofol attenuated intestinal epithelial apoptosis after I/R, which was attributed to its antioxidant properties [21]. Similarly, our animals were pre-treated with sedative doses of propofol prior to the induction of cardiac arrest and CPR, which may have significantly alleviated oxidative stress, intestinal injury, and thus BT, suggesting a preconditioning-like effect of propofol [7,16,22]. In addition, it is important to remember that healthy intestinal mucosa expresses low concentrations of tolllike receptor 4 (TLR-4), which is significantly increased in inflammatory conditions [23]. However, propofol has been reported to down-regulate TLR-4 expression, therefore delaying the reperfusion-induced BT [24]. Further studies in different animal models and particularly in the clinical setting are warranted to determine if this response translates to the bedside when propofol is used during the peri-arrest period.

Although research has shown that the reperfused intestine is characterized by severe histological alterations, mucosal rupture, and increased permeability [23], we did not find significant differences in the intestinal mucosal injury score between the three groups. Our results are similar to those of Schroeder et al. who reported a mild intestinal barrier damage and mild local intestinal inflammation within $24 \mathrm{~h}$ post-ROSC in a rat model of six-minute cardiac arrest [25]. The mild intestinal injury in the CA-CPR group may be also explained by the low dose of the administered adrenaline, which may have minimized the detrimental effect of the drug on mesenteric blood flow [26]. Also, the non-significant differences in endotoxin levels between the CA and the CON group imply that the ischaemic-induced intestinal injury was not able to cause BT. On the other hand, reperfusion of the intestine after CPR and ROSC induced BT, significantly increasing endotoxin levels in the portal circulation of the CA-CPR group [27]. Of note, research has shown that endotoxin increases in more than 24 hours after ROSC, implying that the short monitoring period of 24 hours may be the reason for the non-significant differences in systemic endotoxin levels in our study [27]. Another explanation for this finding may be the circulation of endotoxin, which either translocates through the portal venous system to the liver or enters the systemic circulation via the thoracic duct. Then, a significant fraction of endotoxin binds to plasma proteins and is removed by certain organs, particularly the liver [28].

Although our findings imply that reperfusion may play a causative role in intestinal injury, possibly due to overproduction of oxygen radicals and/or insufficient antioxidant activity, the difference in MDA concentration in ileum and colon may be inversely correlated with the amount of bacteria species and populations commonly hosted in these two sites of the gut. However, gut-derived BT may not be the sole mechanism of post-resuscitation inflammation and sepsis. It is possible that bacteria, macromolecules, immune cells, and cytokines travel along the mesenteric lymphatics to the systemic circulation, entering pulmonary circulation and inducing acute lung injury and multiple organ dysfunction [29]. As the gut-portal 
and the gut-lymph mechanism may occur independently of each other, further studies are required for the elucidation of these phenomena.

Limitations to our study should be considered. First, our experiment was conducted on apparently healthy pigs with no underlying disease. This is not the case in human cardiac arrest victims who, most of the time, have various comorbidities. Second, the monitoring period of the CA-CPR group was limited to 24 hours and different results could be obtained in longer monitoring periods. Due to the small sample size of our study, statistical analysis may have failed to capture other factors associated with BT. Moreover, we did not search for TLR-4 polymorphisms, although some of them may prevent the deleterious effect of endotoxin [30]. In addition, we did not perform repeated measurements of endotoxin. Nevertheless, all measurements were performed at 24 hours following ROSC.

\section{Conclusions}

This is the first study presenting direct evidence regarding the timing of BT after cardiac arrest. Malondialdehyde content and portal endotoxin levels increased significantly only after CPR and ROSC. Although the number of positive MLNs cultures was greater in the CA-CPR animals, no statistically significant differences were observed between the three groups at 24 hours. Future studies on BT should include a post-reperfusion monitoring period of more than 48 hours.

\section{Additional Information \\ Disclosures}

Human subjects: Consent was obtained by all participants in this study. Animal subjects: The study protocol was approved by the Greek General Directorate of Veterinary Services and was conducted in accordance to the Greek legislation regarding ethical and experimental procedures. Issued protocol number 4978/16-10-2015. Conflicts of interest: In compliance with the ICMJE uniform disclosure form, all authors declare the following: Payment/services info: All authors have declared that no financial support was received from any organization for the submitted work. Financial relationships: All authors have declared that they have no financial relationships at present or within the previous three years with any organizations that might have an interest in the submitted work. Other relationships: All authors have declared that there are no other relationships or activities that could appear to have influenced the submitted work.

\section{Acknowledgements}

The authors would like to thank Zacharioudaki, E. Karampela, K. Tsarea, M. Karamperi, N. Psychalakis, A. Karaiskos, and S. Gerakis, staff members of the ELPEN Experimental-Research Centre, for their assistance during the experiments.

\section{References}

1. Berdowski J, Berg RA, Tijssen JG, Koster RW: Global incidences of out-of-hospital cardiac arrest and survival rates: systemic review of 67 prospective studies. Resuscitation. 2010, 81:1479-1487. 10.1016/j.resuscitation.2010.08.006

2. Girotra S, Nallamothu BK, Spertus JA, Li Y, Krumholz HM, Chan PS: Trends in survival after in-hospital cardiac arrest. N Engl J Med. 2012, 367:1912-1920. 10.1056/NEJMoa1109148

3. Chalkias A, Xanthos T: Post-cardiac arrest syndrome: Mechanisms and evaluation of adrenal insufficiency . World J Crit Care Med. 2012, 1:4-9. 10.5492/wjccm.v1.i1.4

4. Coba V, Jaehne AK, Suarez A, et al.: The incidence and significance of bacteremia in out of hospital cardiac arrest. Resuscitation. 2014, 85:196-202. 10.1016/j.resuscitation.2013.09.022

5. Chalkias A, Scheetz MH, Gulati A, Xanthos T: Periarrest intestinal bacterial translocation and resuscitation outcome. J Crit Care. 2016, 31:217-220. 10.1016/j.jcrc.2015.09.015

6. Tassopoulos A, Chalkias A, Papalois A, Iacovidou N, Xanthos T: The effect of antioxidant supplementation on bacterial translocation after intestinal ischemia and reperfusion. Redox Rep. 2017, 22:1-9. 10.1080/13510002.2016.1229893

7. Hsing $\mathrm{CH}$, Lin MC, Choi PC, et al.: Anesthetic propofol reduces endotoxic inflammation by inhibiting reactive oxygen species-regulated Akt/IKKß/NF-KB signaling. PLoS One. 2011, 6:17598. 10.1371/journal.pone.0017598

8. Raffay V, Chalkias A, Lelovas P, et al.: Addition of glucagon to adrenaline improves hemodynamics in a porcine model of prolonged ventricular fibrillation. Am J Emerg Med. 2014, 32:139-143. 10.1016/j.ajem.2013.10.030

9. Papalexopoulou K, Chalkias A, Pliatsika P, et al.: Centhaquin effects in a swine model of ventricular fibrillation: centhaquin and cardiac arrest. Heart Lung Circ. 2017, 26:856-863. 10.1016/j.hlc.2016.11.008

10. Chalkias A, Spyropoulos V, Koutsovasilis A, Papalois A, Kouskouni E, Xanthos T: Cardiopulmonary arrest and resuscitation in severe sepsis and septic shock: a research model. Shock. 2015, 43:285-291. 10.1097/SHK.0000000000000285

11. Kontouli Z, Staikou C, Iacovidou N, et al.: Resuscitation with centhaquin and $6 \%$ hydroxyethyl starch 130/0.4 improves survival in a swine model of hemorrhagic shock: a randomized experimental study. Eur J Trauma Emerg Surg. 2019, 45:1077-1085. 10.1007/s00068-018-0980-1

12. Dragoumanos V, Iacovidou N, Chalkias A, et al.: Passive leg raising during cardiopulmonary resuscitation 
results in improved neurological outcome in a swine model of prolonged ventricular fibrillation. Am J Emerg Med. 2012, 30:1935-1942. 10.1016/j.ajem.2012.04.014

13. Monsieurs KG, Nolan JP, Bossaert LL, et al.: European Resuscitation Council guidelines for resuscitation. Section 1. Executive summary. Resuscitation. 2015, 95:1-80. 10.1016/j.resuscitation.2015.07.038

14. Swindle MM, Volger GA, Fulton LK, Marini RP, Popilskis S: Preanaesthesia, anesthesia, analgesia and euthanasia. Laboratory Animal Medicine, 2nd ed. Fox JG, Anderson LC, Loew FM, Quimby FW (ed): Academic Press, New York; 2002. 955-1003. 10.1016/B978-012263951-7/50025-9

15. Lewis D: Animal experimentation: Implementation and application of the 3Rs . Emerg Top Life Sci. 2019, 3:675-679. 10.1042/ETLS20190061

16. Liu KX, Rinne T, He W, Wang F, Xia Z: Propofol attenuates intestinal mucosa injury induced by intestinal ischemia-reperfusion in the rat. Can J Anaesth. 2007, 54:366-374. 10.1007/BF03022659

17. Smith AJ, Lilley E: The role of the three Rs in improving the planning and reproducibility of animal experiments. Animals (Basel). 2019, 9:975. 10.3390/ani9110975

18. Ozkan OV, Yuzbasioglu MF, Ciralik H, et al.: Resveratrol, a natural antioxidant, attenuates intestinal ischemia/reperfusion injury in rats. Tohoku J Exp Med. 2009, 218:251-258. 10.1620/tjem.218.251

19. Chalkias A, Mamais I, Xanthos T: Microcirculation-mediated preconditioning and intracellular hypothermia. Med Hypotheses. 2018, 115:8-12. 10.1016/j.mehy.2018.03.006

20. Chalkias A, Xanthos T: Redox-mediated programed death of myocardial cells after cardiac arrest and cardiopulmonary resuscitation. Redox Rep. 2012, 17:80-83. 10.1179/1351000212Y.0000000002

21. Liu KX, Chen SQ, Huang WQ, Li YS, Irwin MG, Xia Z: Propofol pretreatment reduces ceramide production and attenuates intestinal mucosal apoptosis induced by intestinal ischemia/reperfusion in rats. Anesth Analg. 2008, 107:1884-1891. 10.1007/BF03022659

22. Vasileiou I, Kalimeris K, Nomikos T, et al.: Propofol prevents lung injury following intestinal ischemiareperfusion. J Surg Res. 2012, 172:146-152. 10.1016/j.jss.2010.07.034

23. Arumugam TV, Okun E, Tang SC, Thundyil J, Taylor SM, Woodruff TM: Toll-like receptors in ischemiareperfusion injury. Shock. 2009, 32:4-16. 10.1097/SHK.0b013e318193e333

24. Wu GJ, Chen TL, Chang CC, Chen RM: Propofol suppresses tumor necrosis factor-alpha biosynthesis in lipopolysaccharide-stimulated macrophages possibly through downregulation of nuclear factor-kappa Bmediated toll-like receptor 4 gene expression. Chem Biol Interact. 2009, 180:465-471. 10.1016/j.cbi.2009.05.003

25. Schroeder DC, Maul AC, Mahabir E, et al.: Evaluation of small intestinal damage in a rat model of 6 minutes cardiac arrest. BMC Anesthesiol. 2018, 18:61. 10.1186/s12871-018-0530-8

26. Korth U, Krieter H, Denz C, et al.: Intestinal ischaemia during cardiac arrest and resuscitation: Comparative analysis of extracellular metabolites by microdialysis. Resuscitation. 2003, 58:209-217. 10.1016/s03009572(03)00119-9

27. Grimaldi D, Sauneuf B, Guivarch E, et al.: High level of endotoxemia following out-of-hospital cardiac arrest is associated with severity and duration of postcardiac arrest shock. Crit Care Med. 2015, 43:25972604. 10.1097/CCM.0000000000001303

28. Munford RS: Endotoxemia-menace, marker, or mistake?. J Leukoc Biol. 2016, 100:687-698. 10.1189/jlb.3RU0316-151R

29. Chalkias A, Xanthos T, Papageorgiou E, Anania A, Beloukas A, Pavlopoulos F: Intraoperative initiation of a modified ARDSNet protocol increases survival of septic patients with severe acute respiratory distress syndrome. Heart Lung. 2018, 47:616-621. 10.1016/j.hrtlng.2018.06.011

30. Arbour NC, Lorenz E, Schutte BC, et al.: TLR4 mutations are associated with endotoxin hyporesponsiveness in humans. Nat Genet. 2000, 25:187-191. 10.1038/76048 